\title{
Les tensions ethniques dans les familles péruviennes coloniales
}

Réalités et/ou alibis? (xvII ${ }^{e}$ et $\mathrm{XVIII}^{\mathrm{e}} \mathrm{s}$.)

\section{Bernard Lavallé}

\section{(2) OpenEdition}

Journals

\section{Édition électronique}

URL : http://journals.openedition.org/clio/7461

DOI : $10.4000 /$ clio.7461

ISSN : 1777-5299

Éditeur

Belin

Édition imprimée

Date de publication : 1 janvier 2008

Pagination : 135-151

ISBN : 978-2-85816-973-3

ISSN : 1252-7017

Référence électronique

Bernard Lavallé, "Les tensions ethniques dans les familles péruviennes coloniales », Clio. Histoire, femmes et sociétés [En ligne], 27 | 2008, mis en ligne le 23 août 2013, consulté le 21 avril 2019. URL http://journals.openedition.org/clio/7461 ; DOI : 10.4000/clio.7461

Ce document a été généré automatiquement le 21 avril 2019.

Tous droits réservés 


\title{
Les tensions ethniques dans les familles péruviennes coloniales
}

\author{
Réalités et/ou alibis ? (XVII ${ }^{\mathrm{e}}$ et XVIII ${ }^{\mathrm{e}} \mathrm{s}$ )
}

\section{Bernard Lavallé}

1 Travailler sur la famille et les relations de couple en Amérique coloniale, ne peut se faire sans se confronter aux questions ethniques. Il ne pouvait, sans doute, en être autrement dans cette société multiethnique dans laquelle le métissage était devenu une donnée essentielle.

2 Toutefois, la réalité était beaucoup plus compliquée que ne pourrait le laisser penser un examen rapide de ce genre de situations. Les différences ethniques, ou réputées telles, pouvaient avoir dans l'histoire des couples des «fonctions » diverses, et être l'objet de bien des manipulations.

3 Le mariage étant un sacrement, tous les problèmes pouvant survenir lors de sa célébration ou par la suite dans la vie du couple, lorsqu'ils pouvaient de par leur gravité entraîner un différend majeur voire une séparation, devaient être soumis aux tribunaux ecclésiastiques qui les traitaient comme de véritables procès. Les archives épiscopales conservent de ce fait une masse considérable de dossiers qui constituent une source à peu près unique sur les relations conjugales vues de l'intérieur, et donc un observatoire privilégié quant à la nature et à la portée réelle de possibles incidences dans la vie des couples des règles socio-raciales - non écrites pour la plupart, mais bien réelles néanmoins - qui prévalaient dans la société coloniale hispano-américaine. Les cas étudiés dans cet article concernent les $\mathrm{XVII}^{\mathrm{e}}$ et $\mathrm{XVIII}^{\mathrm{e}}$ siècles. Les archives proviennent du monde andin - des archives de Lima et de Quito, épiscopales pour l'essentiel de la période, nationales pour sa fin. Mais leur valeur signifiante dépasse probablement de beaucoup le cadre qui les a vu naître. 


\section{L'argument de la desigualdad dans les demandes d'annulation de mariage}

Dans les dossiers concernant des problèmes de couples soumis à l'examen et au jugement des tribunaux ecclésiastiques, il n'est pas rare d'observer que les questions ethniques se trouvent au cœur des problèmes. Ainsi, dans les demandes d'annulation de mariage, on constate souvent que les parents sont intervenus de façon décisive afin de ne pas déroger à l'ordre socio-racial de l'édifice colonial. Quelques exemples? En 1667, la mulâtresse Bernavela de Aguilar, qui avait épousé un sergent du nom de Felipe Claros, déclara, sous la plume de son avocat, que ses difficultés conjugales avaient eu pour origine sa bellemère. Celle-ci n'avait pas supporté que "étant mulâtresse » disait Bernavela - «je me marie avec son fils ». Par des insinuations et de méchantes remarques, la belle-mère avait créé une situation qui s'était envenimée et n'avait pas tardé à devenir insupportable :

Ledit Felipe Claros, irrité par les regrets de sa mère et de son frère, par les humiliations et les affronts qu'ils me faisaient subir parce qu'il m'avait épousée, moi une mulâtresse, a fini par me détester ${ }^{1}$.

5 L'espoir de beaucoup de familles était de pouvoir agir de sorte que leurs enfants, en général leurs filles, puissent se marier avec quelqu'un situé plus haut qu'elles dans la "hiérarchie ethnique» de ce que certains ont pu appeler une société "pigmentocratique ». Cette variante de la mobilité sociale, était connue sous l'expression mejorar la raza (améliorer la race) variante hispano-américaine du sauver la peau des Antilles françaises.

Pour arriver à leurs fins, ces familles n'hésitaient pas à employer parfois des méthodes pour le moins discutables, ce qui ouvrait ensuite la possibilité à des plaintes en justice. Alors qu'il était tout jeune, D. Diego de Herrera y Moscoso (le nom à lui seul indique de quelle strate sociale il provenait), fils d'un militaire de haut rang (maestre de campo) bien apparenté, avait fui de chez sa mère qui voulait l'obliger à être prêtre. Diego s'était alors caché dans une famille dont la mère était zamba, métisse d'Indien et de Noir. Peu après, étant donné son jeune âge, il s'était laissé convaincre et avait fini par se marier avec l'une des filles de la maison, mais sous une fausse identité. Plus tard, devenu un homme, il demanda l'annulation de son mariage en arguant qu'étant espagnol, donc blanc, le fait d'avoir épousé une fille de zamba suffisait à prouver que sa décision n'avait pas été libre, ou en tout cas n'avait pas été prise en connaissance de cause.

On ne peut croire que, si je ne m'étais pas trouvé dans une telle gêne et si j'avais eu le temps de considérer les obligations de mon sang, j'aurais consenti à cette union avec la fille d'une zamba. Il en résulte manifestement que je n'ai pas agi librement et spontanément ${ }^{2}$.

7 Le contraire pouvait aussi exister, lorsque des familles désireuses d'éviter des difficultés ou les désillusions essayaient d'empêcher des unions qui auraient produit un déséquilibre apparemment favorable, mais à la longue peut-être problématique. Isabel Francisca de Chaves, fille métisse d'un corregidor de Yauyos mais qui vivait avec sa mère indienne, voulait se marier avec un Espagnol dont elle avait déjà deux enfants. La mère, craignant que cet homme ne soit intéressé que par les biens de sa fille, avait obligée celle-ci à épouser un Indien mitayo (de corvée). Comme l'écrivait Isabel, sa mère avait pensé que «ce serait pour mon plus grand avantage parce qu'il se conduirait comme mon domestique, ne maltraiterait pas ma mère et ne dissiperait pas mes biens ». La jeune 
femme s'était opposée à cette union, mais en vain, car, écrivait-elle, «je considérais dévalorisant d'épouser un Indien de corvée alors que j'étais métisse et mes enfants espagnols, blancs et blonds $»^{3}$.

Parmi les demandes d'annulation de mariage figuraient aussi des cas de violences physiques exercées pour contraindre une personne située plus haut dans la hiérarchie " socio-raciale ». Les victimes en étaient presque toujours de jeunes femmes. Gertrudis de Melgar, qui se disait espagnole, raconte comment son professeur de harpe, l'Indien Melchor de los Reyes, l'avait aidée à fuir de chez ses parents. Ensuite, il l'aurait enfermée chez lui, puis violée, l'obligeant ainsi à se marier avec lui sous peine, si elle refusait, de tout révéler au grand jour. Gertrudis avait fini par accepter de se marier, mais de façon clandestine et à contrecœur « vu qu'il s'agissait d'une personne inférieure puisqu'il était indien $»^{4}$.

9 Les hommes pouvaient aussi être victimes, parfois, de cette volonté de blanchiment matrimonial. Le Navarrais Felipe de Vera était l'amant d'une métisse, Rosa de Espinosa qui le voulait pour mari. Comme il refusait, elle le fit emprisonner et le menaça de le faire exiler à Valdivia, dans le sud chilien, dont l'isolement et le froid servaient d'épouvantail aux Liméniens. Le mariage finit par avoir lieu, mais de manière tout à fait irrégulière. Lorsque le plaignant demanda son annulation, pour preuve du piège où il était tombé, il affirma que «... la personne n'étant pas mon égale et ladite Rosa de Espinosa une métisse, dont le sang ne valait pas le mien, je ne devais pas épouser une personne qui ne serait pas mon égale $»^{5}$.

10 En 1666, Bartolomé López de Chaves, Espagnol demeurant à Huancayo, demanda l'annulation de son mariage, affirmait-il, car il s'était marié douze ans auparavant avec une Indienne, Francisca Guarmitae, à la suite de "ses tromperies »"

11 Pareilles préoccupations à propos de l'inégalité réelle ou supposée sur le plan ethnique, ne concernaient pas seulement des Espagnols ou des métis d'un certain rang. Parmi les plaignants ou les plaignantes tous les milieux sont représentés, toutes les positions sociales, tous les groupes ethniques, ce qui prouvait bien le caractère généralisé de ces préjugés et des problèmes qu'ils soulevaient. En 1664, Pascuala María, zamba libre, dénonçait que sa maîtresse l'avait mariée de force onze ans plus tôt à « un esclave noir récemment arrivé d'Afrique ", un bozal. Pascuala insistait sur le fait qu'elle était libre et «seulement » mulâtresse. Cela suffisait à prouver à ses yeux que «si on l'avait laissée libre d'agir librement, elle ne l'aurait pas fait $»^{7}$. En 1680, l'esclave créole, donc née au Pérou, Dominga Juana, qui appartenait aux Jésuites, demanda l'annulation de son mariage auquel les Pères l'avaient contrainte. Ils pensaient ainsi mieux retenir le mari, un nègre marron, voleur « et ayant bien d'autres défauts » le premier étant aux yeux de Dominga qu'il était bozal...8.

On trouve aussi des situations de ce type en milieu indigène. La métisse Leonor Álvarez s'était mariée sept ans plus tôt, dans l'église des Espagnols de Ica, en imaginant que son fiancé était lui aussi métis. Elle déchanta lorsqu'elle se rendit compte qu'elle s'était unie à un Indien, et elle exigea l'annulation de son mariage, en expliquant les raisons de sa frustration:

Il est certain que je ne me serais pas mariée avec le susdit parce que je ne veux pas que mes enfants, nés ou à naitre, soient tributaires, et eux ne veulent pas être assujettis aux juges et aux curés qui nous obligeaient à aller à l'église, à la messe pour écouter la doctrine, et bien d'autres choses encore auxquelles les Indiens et les Indiennes doivent se soumettre? 


\section{Les problèmes des promesses de mariage non tenues} femmes, environ un quart provenait de ce que l'homme, qui n'avait eu aucun problème à avoir des relations sexuelles avec les plaignantes, refusait ensuite de tenir parole, en raison de l'origine ethnique de leur maîtresse. L'Église considérant les verba de futuro comme engagement et la première étape vers le mariage, il fallait donc son jugement en la matière pour décider de la suite à donner à pareilles situations.

En 1717, Ana María de Carvajal dénonça Juan de Rivera y Palos. Il s'agissait d'un soldat né dans la Péninsule, et désireux désormais de se marier avec une Espagnole une « jeune fille de vertu reconnue ", alors que Ana María, selon ce que lui faisait dire son avocat, n'était qu'« une zamba de couleur». Juan de Rivera organisait sa défense autour de divers arguments dont le principal était le suivant: «On ne peut croire - écrivait-il - qu'un Espagnol puisse promettre le mariage à une mulâtresse vieille et laide qui n'était ni vierge ni riche. Il faut donc en conclure qu'aucun intérêt ne me poussait à lui faire la promesse supposée $»^{10}$.

de Palacios, qui se disait zamba libre, se présenta devant le tribunal ecclésiastique pour s'opposer au mariage d'Agustín Lozano, né en Espagne. Il refusait de tenir la promesse de mariage qu'il avait faite à la plaignante et voulait maintenant épouser une métisse, Josefa Andrade. Agustín Lozano ne niait pas avoir eu des relations avec la plaignante, mais expliquait qu'il ne pouvait en aucune façon avoir fait avec elle le projet qu'elle prétendait. Il en donnait sans ménagement les raisons :

Je n'ai pas voulu gêner ladite esclave en insistant sur ses défauts mais, maintenant, l'injuste procès pour promesse rompue m'oblige à présenter des témoins à la conduite honnête pour attester de mon honnêteté.

arguments étaient, pensait-il, sans réplique. Le principal, et à ses yeux suffisant, était que Juana de Dios n'était pas libre comme elle le prétendait, mais esclave travaillant à la journée en ville pour sa maîtresse, une religieuse. En outre, il était de notoriété publique lorsqu'ils s'étaient rencontrés que c'était « une femme facile avec deux enfants ». Sans compter que le statut militaire interdisait aux soldats de se marier avec une personne "qui n'était ni libre ni de même couleur " ${ }^{11}$.

Un autre procès intéressant fut celui qui en 1715 opposa Fernando de la Cámara y Campo à Josefa Vélez. Lui était natif de Burgos « noble hidalgo chevalier d'un lignage connu » et résidait à Huaraz où son oncle était corregidor. Elle affirmait que "pour avoir une relation illicite ", D. Fernando l'avait poussée à quitter sa maison familiale et lui avait promis le mariage devant un crucifix. L'accusé donnait une autre version : la famille de la jeune fille l'avait attiré chez elle et l'avait en quelque sorte piégé :

Voyant le champ libre, j'ai commencé à lui manifester une affectueuse soumission qu'elle me rendait bien [...] les caresses amoureuses de la susdite suscitèrent ma passion et, d'un commun accord, nous avons fait ce que fait l'homme tenaillé par le désir, excité par les soins d'une femme.

Josefa avait appris que son ancien amant voulait épouser la fille d'un capitaine, ce qu'il ne niait pas, mais il centrait sa défense autour de deux arguments: les avances qui lui avaient été faites et surtout sur l'inégalité existant entre eux («quand bien même la raison empêcherait de croire qu'elle avait sollicité mon amitié, parce que c'est une attitude impropre d'une femme, la raison régit l'inégalité notoire entre mes devoirs et les 
siens »). On disait en effet que Josefa était la fille d'un prêtre et d'une " métisse pauvre » qui avait eu deux enfants de pères différents. Il est à souligner que le tribunal ne fut pas très convaincu par la défense de l'accusé. Elle lui donna dix jours pour se marier avec Josefa, faute de quoi il devrait lui payer 800 pesos plus les frais de justice. Fernando fit appel de la sentence au motif que la plaignante ne « valait pas » une dot aussi élevée ${ }^{12}$.

\section{La reconnaissance officielle de la notoria desigualdad}

Très tôt, le problème de la notoire inégalité (notoria desigualdad) entre deux jeunes gens était devenu un argument de poids pour faire empêcher un mariage, et bien que rien ne vienne légalement l'étayer, les familles y recouraient fréquemment. Ainsi, en 1745 , le lieutenant de cavalerie Francisco Falconí de Ríos fit emprisonner au Callao un certain Clemente Araujo qui avait promis le mariage à sa fille Micaela âgée de seize ans. Le lieutenant qualifiait Clemente d'Indien et le présentait comme « un homme perdu dont les mauvaises habitudes et les vices sont connus ", mais il insistait en premier lieu sur son « inégalité » par rapport à sa fille, raison pour laquelle si cette union avait eu lieu, on aurait $\mathrm{pu}$ en attendre "qu'amertume et scandale à l'encontre de l'honnête fin du mariage ». Quelque temps plus tard, la jeune Micaela, décillée ou convaincue par les raisons de sa famille, écrivit aux juges pour dénoncer « les fallacieuses persuasions » de Clemente et le fait qu'elle s'était engagée «dans l'élan de son jeune âge ». Quant aux origines de l'accusé, écrivait-elle :

En réponse au soupçon d'être Indien, il dit qu'il ne l'est pas, et que moi et mon père nous devons prouver notre hidalguía, alors que notre réputation d'Espagnols et la sienne d'Indien suffisent largement pour que l'inégalité une fois reconnue, invalide quelque consentement que j'aie pu donner.

Pour sa part, Araujo, natif de Guayaquil, répondit qu'il était «pur, blanc, métis d'Espagnol » et que sa mère était « une métisse pure ». Les juges ne furent pas convaincus et il n'obtint pas gain de cause ${ }^{13}$.

Cette question de la notoria desigualdad enracinée dans les pratiques sinon dans la loi, donnait souvent lieu à des débats intéressants, car les personnes auxquelles on l'opposait ne manquaient pas de réagir et de défendre leur point de vue, où transparaît en clair ou en filigrane, une conception des nuances ethniques et de la hiérarchie qui en découlait sensiblement différente de ce que les règles communément admises pourraient laisser croire.

En 1776, María Asensión Ninavilca y Pizarro «Indienne noble » se présenta devant le tribunal au nom de sa fille, Toribia Landaburu « fille d'un Espagnol et d'une Indienne noble, jouissant de tous les privilèges que sa Majesté octroie à la Nation ». Toribia s'était promise à Marcos Fernández, mais le beau-frère de ce dernier, un officier, s'opposait au mariage sous prétexte que Marcos «se rabaissait». Dénonçant le fait que sa fille fût traitée d'Indienne, María Asensión répliquait avec orgueil :

Je suis une Indienne de noble extraction, issue d'une famille de caciques, comme cela est notoire et comme je pourrais le prouver sur le champ. Le père de ma fille, don Miguel de Landaburu, est un homme d'honneur, distingué par son poste.

Quant aux proches de Marcos, selon la plaignante, ils n'avaient aucune raison d'être aussi orgueilleux. Ils étaient adultérins et élevés dans une famille qui vivait dans « une ruelle en compagnie de prostituées qui donnaient le mauvais exemple à ses filles » ${ }^{14}$. 

niveau social différents, ce qui constituait un risque pour «l'ordre social et provoquait des frictions nuisibles et des préjudices continuels dans les familles ». On voulait ainsi «contenir l'anarchie qui, au fil du temps, s'était infiltrée lentement dans la société ». L'union inégale «défi [ait] l'honneur, le respect et l'obéissance que les enfants doivent à leur père dans des affaires d'une telle importance ». L'État considérait de sa responsabilité le contrôle - et l'interdiction - de pareils mélanges, et il donnait aux familles l'appareil juridique pour s'interposer. Les parents ou les tuteurs, dont l'accord était obligatoire pour les mineurs de moins de 25 ans, pourraient donc s'appuyer sur ce texte, et l'on aurait le droit de déshériter quiconque se marierait contre l'avis de sa famille. Désormais, outre les empêchements canoniques en vigueur, il suffirait de démontrer l'inégalité entre deux futurs conjoints pour que le mariage ne puisse avoir lieu.

Deux ans plus tard, le 7 avril 1778, ce texte fut étendu à l'Empire. En Espagne, les nouvelles dispositions étaient applicables à tous les sujets «sans exception aucune, depuis les hautes classes jusqu'aux plus basses ». En Amérique, les unions ne concernant que des mulâtres, des métis ou des Noirs « et d'autres races mixtes » seraient cependant 
exclues. La Pragmatique qui, dans la Péninsule, était de nature fondamentalement sociale, en Amérique devenait un moyen de "protéger » les familles blanches de mélanges non désirés, et il s'agissait de restreindre une évolution toujours plus forte vers le métissage. Par la suite, les dispositions de 1778 furent plusieurs fois précisées, mais toujours dans un sens de renforcement restrictif et afin de donner aux représentants de l'État et aux parents plus de pouvoirs.

Nous ne nous étendrons pas sur les procédures engagées dans la mesure où nous leur avons déjà consacré une étude dans le cas quiténien ${ }^{16}$. Simplement, lorsque de telles procédures étaient engagées par les familles, presque toujours en brandissant l'argument d'ancêtres noirs parmi ceux du conjoint refusé, ces accusations ne restaient pas sans réponse chez les personnes concernées. Celles-ci se défendaient en essayant de démontrer que ce qu'on leur reprochait était faux, mais aussi en fouillant dans l'ascendance de la partie adverse, et en prouvant qu'elle était mal fondée à discuter d'une éventuelle inégalité puisqu'on pouvait lui rappeler que ses ancêtres n'étaient pas aussi blancs qu'elle le prétendait. On trouvait toujours quelque métisse, voire quelque indienne ou une mulâtresse dans l'arbre généalogique dont on pouvait utiliser opportunément le souvenir. On peut voir ainsi en filigrane les stratégies de blanchiment de bien des familles qui n'entendaient pas perdre leur bénéfice par la faute d'un mariage à leurs yeux bien intempestif.

Ces réactions montrent aussi qu'en cette fin de XvIII siècle, la réalité ethnique était bien souvent occultée, voire oubliée au bout de quelques générations, par l'ascension sociale. Quant aux personnes accusées, leur défense révèle que l'inégalité ethnique n'était plus pour eux un tabou, que les moins favorisés à cet égard avaient désormais une conscience très claire de sa portée et de son sens très relatifs.

Dans la plupart de ces dossiers, on voit bien la présence des préjugés ethniques et la confirmation de leur prégnance dans la vie quotidienne de la colonie. Cependant, le fait que certaines familles utilisent l'argument de l'inégalité pour empêcher le mariage de leur enfant montre aussi, de manière en quelque sorte paradoxale, que les compartiments n'étaient pas si étanches. Les individus mus par l'amour, ou l'intérêt, pouvaient franchir, voire abolir les barrières invisibles mais bien réelles de la pigmentocratie.

\section{De bien étranges ignorances...}

On a vu que ceci était une réalité bien avant que la Pragmatique de 1778 n'intervienne de manière officielle. D'autres considérations peuvent nous conduire à moduler l'appréhension que l'on pourrait avoir du phénomène au terme d'une lecture superficielle de la documentation. D'abord, dans ce genre de dossier, l'attention est retenue par l'aspect confus, et l'expression imprécise des réalités ethniques. Dans cette société bigarrée, dans laquelle les marqueurs ethniques jouaient un rôle aussi important, il est surprenant que certains individus aient essayé - et apparemment réussi pendant un certain temps, voire de longues années - à se faire passer pour des Blancs, ou en tout cas pour plus blancs qu'ils n'étaient vraiment. De tels faits pouvaient donner lieu à des situations difficiles et compliquées dans lesquelles le conjoint trompé, honteux et frustré, entamait des démarches en vue de faire annuler son mariage. Le plus souvent, il y parvenait lorsqu'il pouvait prouver sa demande quant à l'inégalité qui en résultait et qui avait été frauduleusement cachée. Les juges ecclésiastiques considéraient alors qu'il y avait eu « erreur - il conviendrait de dire tromperie - sur la personne ». 

Jacques, avait épousé Juan de Herrera y Castilla. Il se prétendait « chevalier de grande qualité », unique héritier d'haciendas et d'encomiendas. Cinq ans plus tard, Da. Jerónima sollicita l'annulation de son mariage après s'être rendue compte que sa belle-mère n'était autre que l'Indienne Juana Topachi ${ }^{17}$. En 1667, Damiana Hurtado voulut se séparer de son mari Pedro Hernández. D’après les papiers qu'il avait montrés, il était espagnol et fils d'un capitaine, or, après le "soulèvement " indien de l'année précédente, il avait été arrêté comme métis, et avoua que son père était métis et sa mère indienne ${ }^{18}$. déjà cité de la métisse Leonor Álvarez. Même les esclaves pouvaient demander l'annulation de leur mariage à la suite d'erreur sur la qualité du conjoint. La mulâtresse Inés de escobar voulait être séparée du mulâtre, lui aussi esclave, Antonio Moreno. Son avocat faisait valoir qu'il était violent et alcoolique, mais surtout que «lorsqu'il s'était marié, il avait laissé entendre qu'il était mulâtre alors qu'il est morisque, descendant de Barbaresques et, pour cette raison, ses mœurs sont mauvaises et dépravées et son tempérament est dur $»^{19}$.

Il n'était pas exceptionnel aussi que les esclaves cachent leur condition en se mariant, et lorsqu'on découvrait la réalité, le conjoint tout marri demandait l'annulation de cette escroquerie au mariage. Cela arrivait dans les familles mulâtres, c'est-à-dire des milieux ambigus d'un point de vue social et ethnique puisqu'ils étaient apparentés à des Blancs mais aussi liés au monde de l'esclavage. En outre, les erreurs et les tromperies étaient facilitées par le fait que rien ne ressemblait plus à un mulâtre libre qu'un mulâtre esclave et que certains, après plusieurs générations de métissage pouvaient passer pour blancs.

Cependant, ces «mésaventures " n'épargnaient pas les couches plus aisées. Da. Juana Atienso demanda l'annulation de son mariage avec Cristóbal Muñoz au bout de six mois. Elle était «une Espagnole de parents nobles» et venait de découvrir que son époux, originaire de Trujillo, était esclave dans sa ville natale ${ }^{20}$. La même chose était arrivée à Da. Josefa María Martínez de Soto - elle aussi noble, disait-elle - avec son mari Pedro Ramírez ${ }^{21}$. L'enseigne Martín de Roa avait épousé une mulâtresse orpheline élevée par un maestre de campo. Lorsque celui-ci mourut, son gendre et exécuteur testamentaire révéla au mari que son épouse était en fait la fille adultérine de son tuteur avec une de ses esclaves et, par conséquent, qu'elle «était esclave de la famille». Pour résoudre le problème sans publicité, dommageable pour l'enseigne, mais aussi afin de ne pas léser les héritiers, le gendre proposa à Martín de Roa de lui vendre sa femme pour qu'il puisse ensuite la libérer. L'enseigne s'y refusa et demanda l'annulation du mariage, car lui était désireux de rendre public la tromperie dont il avait été victime et aussi pour n'avoir pas le risque d'avoir des enfants avec une esclave ${ }^{22}$.

Dans de pareils cas, l'erreur sur la personne épousée semble bien manifeste. Cependant d'autres paraissent plus étonnants et posent vraiment question. Josefa de Peralta avait épousé un certain Eugenio de Cantillana en croyant qu'il était espagnol alors qu'il s'agissait d'un quarteron de mulâtre ${ }^{23}$. Comment Ángela de Velasco y Morales avait-elle pu penser que son mari, Damián de Alarcón, en fait un Indien, était, comme il le prétendait, un Alarcón de Panama et espagnol ${ }^{24}$ ? Comment Damiana Hurtado ne s'étaitelle pas rendue compte que Pedro Fernández, qui se disait espagnol, avait une mère indienne et un père métis ${ }^{25}$. On pourrait multiplier les exemples... 
38 Dans une société qui attachait tant d'importance à tous les marqueurs sociaux et ethniques, de telles erreurs paraissent assez étranges, même compte tenu de la complexité et de la multiplicité des mélanges qui, génération après génération, avaient fait que les choses n'étaient pas simples et que les frontières en principe fondées sur l'apparence n'étaient plus aussi claires qu'au début de l'époque coloniale.

39 Sans écarter la possibilité d'erreurs, nous croyons plutôt que dans les situations de ce genre jouait aussi un autre élément. Si de pareilles erreurs ethniques étaient invoquées, c'était sans doute comme un alibi ou pour cacher d'autres raisons, les demandeurs sachant fort bien qu'il y avait là aux yeux des chanoines un argument certes plausible, mais surtout efficace pour obtenir ce qu'ils demandaient, à savoir l'annulation de leur mariage, perspective bien plus intéressante que le divorce puisqu'ils pourraient ensuite se remarier.

Comment Ana Rodríguez avait-elle attendu dix-neuf ans avant de s'apercevoir que son époux était un métis même pas baptisé ${ }^{26}$ Comment Jacinto Rosales n'avait-il pas argué du fait que sa femme était esclave qu'après treize années de vie commune ?27

41 Il y avait sans doute d'autres motifs derrière ceux officiellement donnés. Parfois, cette duplicité apparaît dans les dossiers. C'était, par exemple, des raisons économiques dans le cas de Juan Bazarrate, un mulâtre. Il affirmait avoir ignoré que son épouse, une mulâtresse, était esclave et il centrait là-dessus sa demande. Cependant, un peu plus loin, il disait qu'avant le mariage elle lui avait promis de racheter sa liberté, chose qu'elle n'avait toujours pas faite au bout de quatorze ans de vie commune ${ }^{28}$.

42 Ailleurs les motifs variaient en fonction du moment et de ce que les demandeurs croyaient être plus convaincants aux yeux des juges ecclésiastiques. Bernavela de Aguilar demanda en 1667 l'annulation de son mariage en se plaignant de l'attitude raciste de sa belle-mère qui regrettait l'inégalité de mariage de son fils. N'ayant pas obtenu satisfaction, elle demanda ensuite le divorce à deux reprises, en 1668 et 1670. La première fois, elle donnait comme argument des violences domestiques, la seconde le fait qu'elle aurait eu des relations sexuelles avec un des frères de son mari...

Dans certains dossiers, il arrive même que les demandeurs se repentent d'avoir utilisé des arguments ethniques faux, pour des raisons qu'ils ne précisent pas mais que l'on peut imaginer. En 1682, Juana de Dios de Céspedes retira sa demande d'annulation de mariage où elle avait affirmé que son mari lui avait caché son statut d'esclave. Elle avouait maintenant que cela était faux et qu'elle avait toujours su à quoi s'en tenir à ce sujet ${ }^{29}$.

Pour terminer, nous donnerons un exemple, certes plus tardif, qui représente bien l'adaptabilité des arguments de type racial en fonction des intérêts du moment. En 1803, D. Javier Toredesillas Polido y Escorza, prêtre à Tumbaco près de Quito, s'opposa au mariage de sa cousine Da Ana María Ruiz Polido y Escorza «séduite par un vieil octogénaire du nom de Miguel Almeyda, veuf, très pauvre, chargé d'enfants et réputé zambo ». Alors qu'elle était, au contraire, bien apparentée et même noble, le cousin disait dudit Miguel que «sa couleur le rend suspect [...] sa mère devait être mulâtresse et son père de couleur douteuse ». Sa seconde épouse avait été d'une famille de caciques, et il exerçait le métier bien peu considéré de passeur sur une rivière. Il avait même des arrière-petits-enfants et en plus... était borgne.

45 Après être restée ferme, avoir indiqué que son promis était issu d'un mariage tout à fait décent, et que personne n'avait le droit « d'entraver son absolue liberté de choisir un état vertueux ", Ana María finit par signer une lettre destinée à son cousin où elle 
reconnaissait que « le mariage avec Miguel Almeyda, dans lequel [elle] ne voyai [t] qu'un moyen de trouver quelqu'un pour [la] secourir, satisfaire [s] es besoins et ceux de [s] es enfants, avait occasionné d'horribles hontes». Elle renonça donc à son projet vue «la mauvaise qualité » de son prétendant.

Pourtant, deux années plus tard, Ana María porta plainte contre sa famille. En effet, elle avait accepté de rompre avec Miguel en échange de la promesse d'une rente! Ses cousins n'avaient tenu parole que six mois, puis s'étaient refusés à continuer de payer, en disant qu'Ana María avait du bien. Dans les documents qu'elle signa alors, elle revint sur l'argument de l'origine ethnique de Miguel :

En ce qui concerne la supposée origine inférieure d'Almeyda, je dis que rien n'est prouvé et que les témoins qui ont déposé ont été présentés par D. Xavier Tordesillas [...]. Il est certain que tous sont de misérables paysans dont la naturelle imbécillité peut les conduire à s'engager sottement pour ne pas déplaire à leur curé.

Pour sa part, Ana María n'hésitait pas maintenant à affirmer qu'Almeyda était «un homme d'honneur et de naissance digne $»^{30}$.

Les exemples que nous avons examinés peuvent confirmer la force des préjugés raciaux et leur extension à tout l'éventail social de la colonie, dans la mesure où l'on note à la fois leur caractère récurrent et la variété des milieux concernés. Pratiquement personne n'y échappe, même dans les couches les plus défavorisées de la société. Le mariage apparait bien, souvent pour les femmes et leurs familles, mais aussi parfois pour certains hommes, comme un moyen de s'inscrire dans une mobilité socio-raciale ascendante.

Cependant, si l'on inverse la perspective, ils démontrent aussi, par leur existence même, jusqu'à quel point, très tôt, bien des individus des deux sexes n'avaient pas hésité, pour des motifs très divers - allant de l'intérêt matériel le plus immédiat jusqu'à l'amour sincère - à tenter de s'en émanciper, de transgresser les règles non écrites du mariage entre égaux.

51 Les cas étudiés révèlent aussi que les règles de la reproduction ethnique, apparemment rigides, pouvaient n'être, parfois, que de simples alibis, des arguments a posteriori, d'une grande flexibilité, seulement utilisés le moment venu et dans des plaidoyers pro domo. Les barrières ethniques coloniales, loin de contribuer seulement à la création, ou au renforcement, d'une société de compartiments étanches, ont de fait compliqué les situations et aiguisé la subtilité de bien des aspects des relations humaines.

\section{NOTES}

1. Archivo Arzobispal de Lima (AAL) Nulidades 26 (1667).

2. AAL, Nulidades 45 (1698).

3. AAL, Nulidades 45 (1697).

4. AAL, Nulidades 24 (1664).

5. AAL, Nulidades 45 (1699).

6. AAL, Nulidades 25. 
7. AAL, Nulidades 24.

8. AAL, Nulidades 35.

9. AAL, Nulidades 38 (1684).

10. AAL, Esponsales 8.

11. AAL, Esponsales 10.

12. AAL, Causas criminales de matrimonio 6.

13. AAL, Causas criminales de matrimonio 7.

14. AAL, Esponsales 10.

15. AAL, Esponsales 10.

16. Lavallé Bernard, 1999, « Estrategia o coartada? El mestizaje según los disensos de matrimonio en Quito (1778-1818) », en Amor y opresión en los Andes coloniales, Lima, IFEA-IEP, p. 113-136.

17. AAL, Nulidades 20.

18. AAL, Nulidades 26 (1667).

19. AAL, Divorcios (44) (1670).

20. AAL, Nulidades 29 (1651).

21. AAL, Nulidades 50 (1682).

22. AAL, Nulidades 40.

23. AAL, Lima Nulidades 17 (1650).

24. AAL, Nulidades 34 (1678).

25. AAL, Nulidades 26 (1667).

26. AAL, Nulidades 21 (1661).

27. AAL, Nulidades 45 (1698).

28. AAL, Nulidades 21 (1660).

29. AAL, Nulidades 37.

30. Archivo Nacional del Ecuador, Matrimonios 7.

\section{RÉSUMÉS}

Au moyen de dossiers traités par les tribunaux ecclésiastiques de Lima et Quito, cet article cherche à débusquer les ruses employées pour contourner les règles non écrites de la hiérarchisation socio-raciale de la colonie. Il montre aussi les systèmes de défense des personnes citées à comparaître. Au-delà de schémas somme toute assez répandus dans bien des situations coloniales, on voit apparaître dans la pratique une certaine flexibilité quant à ces règles, les plaignants plaidant souvent la tromperie alors qu'ils semblent bien n'avoir été nullement dupes de l'engagement qu'ils avaient pris avec des personnes situées plus bas qu'eux dans la palette ethnique.

This article brings to light the strategies used to bypass the unwritten rules of the colonial socioracial hierarchy, based on cases reviewed by the ecclesiastical courts of Lima and Quito. It also highlights the defence systems used by people who are appeared in court. In practice, a certain flexibility seems to have existed concerning the rules, a flexibility which went beyond the patterns that are considered common to many colonial situations. In these cases, the plaintiffs often complained that they had been misled when in fact, they often appeared to be fully aware of the engagements they took with people ranked lower in the ethnic hierarchy. 
INDEX

Index chronologique : XVIIIe siècle, XVIIe siècle

Index géographique : Andes

Keywords : Andes 17th and 18th centuries, couple relations, miscegenation, racial prejudice

Mots-clés : métissage, préjugés raciaux, relations de couple

\section{AUTEUR}

\section{BERNARD LAVALLE}

Professeur de civilisation hispano-américaine coloniale à la Sorbonne Nouvelle-Paris III, Bernard Lavallé est spécialiste de la région andine, il est notamment l'auteur d'études sur l'apparition de la conscience créole, l'histoire des relations conjugales et familiales, l'évolution de l'esclavage et le rôle des caciques. 